\title{
IDENTIFICATION OF THE TYPE OF AGRICULTURE SUITED FOR APPLICATION OF WIRELESS SENSOR NETWORKS
}

\author{
Ahsan Abdullah, Ahmed Barnawi, Researchers \\ Faculty of Computing and Information Technology \\ King Abdulaziz University, Jeddah, Saudi Arabia \\ E-mail: aabdullah1@kau.edu.sa
}

Received December 16, 2012

\begin{abstract}
The world's population is expected to double by 2050; world food supply is unlikely to double by doubling the area under cultivation or by doubling the availability of water. There are other challenges too, such as decline in the number of farms and a decline in the number of agriculture workforce. Climate change is expected to further aggravate the existing situation. Therefore, for the humanity to survive agriculture has to become smart - one way is by integrating Wireless Sensor Networks (WSN) in agriculture. In this paper, we will present the application of WSN in agriculture and discuss different types of sensors, different types of WSN and their application in 13 different types of traditional agriculture. We identify the type of agriculture most suited for WSN in terms of applications. We will also review some recent applications of WSN in agriculture; identify challenges and present possible future directions.
\end{abstract}

\section{KEY WORDS}

Smart agriculture; Sensor; Wireless Sensor Network; Monitoring; Cattle monitoring; Greenhouse; Food quality.

\section{Introduction}

Over the last $60+$ years, world agriculture has become significantly effective. There has been an improvement in production systems, availability of fertilizers, high yield varieties etc. Effective livestock management and breeding programs have also contributed. Thus there has been a green revolution and a doubling of food production. However, there are challenges too, such as scarcity of water, pest attacks, and decline in agricultural work force, to name a few. As per FAO (2011) Report, climate change is expected to further aggravate the existing challenges that agriculture faces. As per Burney et al. (2010) and Bruinsma (2009), it is estimated that by 2050 , the current world population will grow from 6.7 billion to 9 billion, with most of the increase occurring in sub-Saharan Africa and South Asia. Considering the changes in the consumption level and composition associated with growing household incomes, it is estimated that feeding the world in 2050 will require a 70 percent increase in total agricultural production.
Objectives of smart agriculture. The objectives of smart agriculture are as follows (www.libelium.com):

1. Using a combination of sensors such as temperature, light and humidity, so as to spot the risk of frost, possible plant diseases and establish watering requirements based on soil dampness.

2. Manage cultivation of crop and to monitor the exact conditions in which the plants are growing from the comfort of your own home.

3 . Control conditions in green houses, nurseries, and closely monitor performance of sensitive crops, such as vines or tropical fruit, where the smallest amount of change in climate can affect the final outcome.

4. Determine the best conditions for each crop, by comparing the data obtained during the best harvests.

Why Smart Agriculture? As per Wark et al. (2007), farming has traditionally been a laborintensive human activity, which involves tending plants and animals on an almost individual basis. However, after the industrial revolution, modern agriculture became automated and mechanized. This has resulted in large areas per farmer and 
subsequent disappearance of small farms. For example, in the US, between 1950 and 1999 the number of farms reduced by 64 percent. In the UK, 200,000 farms vanished between 1966 and 1995, and 17,000 farmers and farmworkers abandoned farming in 2003. Furthermore, the global demographic shift in farm labor and reduced employment of young workers has created an elderly farming population and an impending labor shortage.

The stability of agricultural production is also threatened by climate change. In many areas of the world with agricultural production already on a decline, the means of coping with adversarial events are limited. Climate change is expected to reduce production to even lower levels and make it even more unpredictable (Stern 2006 and Fischer et al. 2002). As part of climate change long term changes in the patterns of precipitation and temperature are expected. These changes are likely to shift production seasons, patterns of pests and diseases. This will subsequently alter the set of feasible crops affecting production, prices, incomes and eventually, livelihoods and lives. So agriculture has to become smarter in order to cope with these climatic challenges; for example sensor-based greenhouse cultivation (section-5.1). As per Tercek (2012), between now and 2050, agriculture has to transform much less territory, increase yields on existing farm and grasslands and use water and other resources more effectively. Fertilizer and pesticides have to be used in a way so as to minimize pollution. All this has to be done while adjusting to fluctuating weather patterns and a more erratic climate. Obviously this strengthening of agriculture has to be sustainable.

As per the US Bureau of Labor Statistics (www.bls.gov), during the 2000-2010 period there has been a decline in agriculture workers and their salaries/wages both for the employed and self-employed. Similar decline is also projected for the 2010-2020 period. Figure-1 shows the percentage change in employment in different domains of Agriculture, projected for 2020. Observe the significant employment increase in technology related Agriculture domains, while the converse being true for manual or labor intensive domains. Thus fueled by the natural and market forces, agriculture is actually on its way to becoming technology centric or "smart".

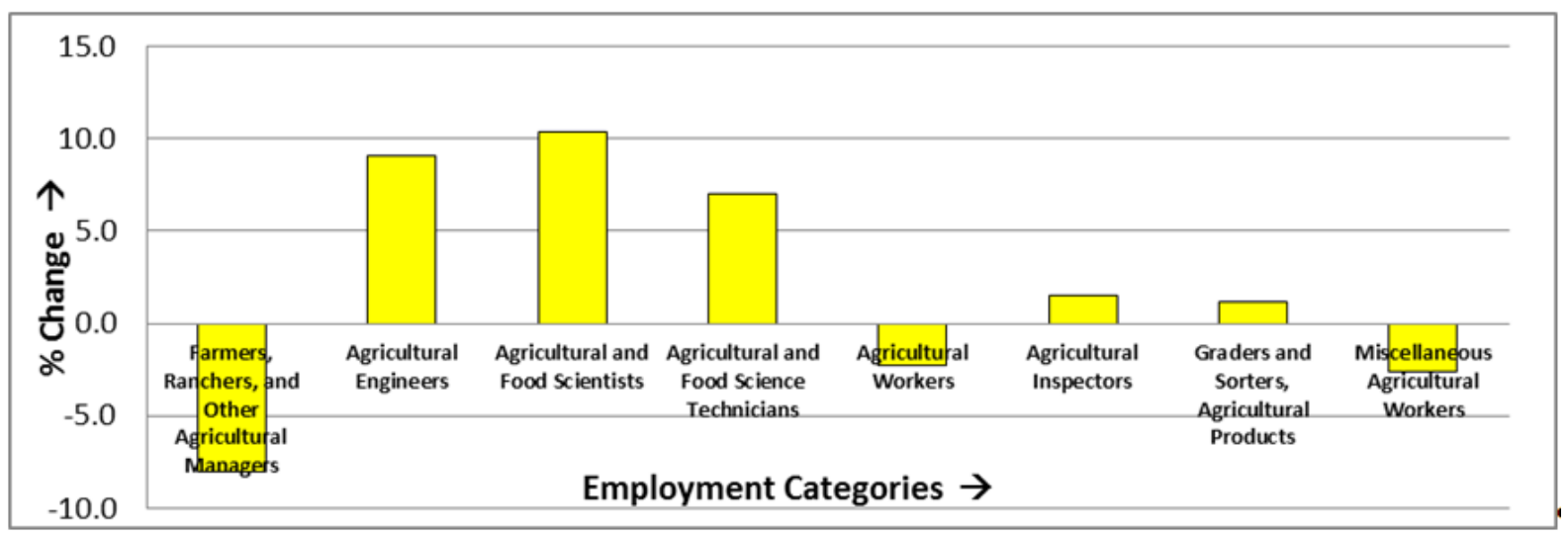

Figure 1 - Projected percentage change in Employment in Agriculture for 2020 (ref: www.bls.gov)

Why Wireless Sensor Networks? As per the MIT Technology Review (2003), Wireless Sensor Networks (WSN) are among the 10 emerging technologies that will change the world; for the better. WSNs are being used in diverse and important applications such as military, agriculture, healthcare, and industrial process monitoring. WSN is an intelligent private network consisting of a large number of sensor nodes having specific functions. Wireless transmission allows the deployment of sensors at remote, dangerous, and harsh environments. Ideally the WSN have number of benefits, such as low power consumption, cost-effectiveness, easy installation, and small size. Wireless sensors can be deployed almost anywhere (including underwater and underground) and that too at a far lower cost as compared to a wired system. As per Thusu (2010), because of the recent developments in wireless technology and embedded systems, the hardware used in WSN has not only become inexpensive, but is also more widely available. These devices 
also comply with industry standards such as the IEEE 802.15.4 for radio communication hardware and the emerging ZigBee and WirelessHART for networking of devices resulting in an increase of end-user adoption.

Rest of the paper is organized as follows. In section-2 classification of sensors is presented along with their current market, in section-3 five types of WSN are discussed, in section-4, 13 types of agriculture are discussed and subsequently compared w.r.t different WSN metrics. In section-5 some recent applications of sensor networks in smart agriculture are discussed which is followed by section- 6 with brief discussion of challenges of sensor networks. Finally possible future directions are presented in section-7.

\section{Why Sensors?}

One of the major problems of agriculture is field data collection and subsequent action. Extensive time and effort is involved in data collection from a large field or plantation. For example, to analyze soil, along with site surrounding temperature, humidity and other field parameters also need to be collected. Field trips could be required every day, or in some cases i.e. for special crops, several times per day in order to collect samples or perform site monitoring. This is not only time consuming, but also requires manpower along with corresponding increase in expenses. Sensors are therefore useful for agriculture data collection and monitoring, especially from the comfort of the farmer's home (Figure-6). As per Miskowicz (2005), over the last few years, event-based sampling and control have received special attention by the researchers from the domain of wireless sensor networks (WSN) and networked control systems (NCS). The reason being, event-based strategies reduce the exchange of information between sensor nodes, controllers, and actuators. This reduction in exchange of information, translates into extension in lifetime of the batterypowered wireless sensors. Reduction in the computational load of the embedded devices, and reduction in the network contention is also achieved.

\subsection{Sensor Classification}

There are literally hundreds and thousands of types of sensors available in the market today, along with different classifications. Some sensor classifications (White, 1987) are based on i) measurement, ii) technology, iii) means of detection, iv) conversion phenomenon, v) sensor material and vi) field of application. The W3C Semantic
Network Sensor incubator group has six classifications of sensors with 69 different principles. For example, measuring humidity using neutron probe, lysimeter, tensiometer etc. In this paper, agriculture sensors are classified by broadly dividing them into three classes based on their properties i.e. i) Physical ii) Mechanical and iii) Chemical. Further breakdown of these sensor types is given in Figure-2, with some of the smart agriculture applications of these sensors discussed in section-5. Note that there can also be other classifications of agriculture sensors, such as i) soil sensor ii) plant sensor iii) weather sensor or sensor classifications based on monitoring vs. tracking and so on.

Each sensor network node (or mote) typically consists of several parts. The main parts being i) a radio transceiver with a connection to an external antenna or an internal antenna, ii) a CPU, iii) an electronic circuit or actuator for interfacing with the sensors and iv) an energy source. The energy source could be a battery or an implanted energy harvester. A biosensor mote is a special miniaturized analytical device, it consists of a biological or biologically derived sensing element which is either connected or integrated within a physicochemical transducer. A sensor node may vary in size from that of a shoebox down to the size of a speck of dust (Park and Chou, 2006).

The motes function within the network and usually fulfill one of the two purposes i) perform data-logging, processing (and/or transmitting) sensor information collected from the environment, ii) or acting as a gateway in the ad hoc wireless network formed by all the sensors. The gateway passes data back to a collection or unique data sink point. Figure-3(a) indicates the functionality oriented relationship of sensors, Fig-3(b) shows the architecture of a conventional wireless sensor node along with typical ratings of its different parts (Chien et al. 2011). Fig-3(b) shows a low-power sensor system, the node being 1,000 times smaller than a comparable conventional commercial counterpart (www.engin.umich.edu).

\subsection{Sensor Market}

The global environmental sensor and monitoring market was grown exponentially over the last decade (www.bccresearch.com) and was valued at $\$ 11.1$ billion in 2010. This market is expected to reach $\$ 15.3$ billion in 2016 i.e. a compound annual growth rate (CAGR) of $6.5 \%$ be- 
tween 2011 and 2016. The market for the terrestrial category is expected to increase at a $5.3 \%$ compound annual growth rate to reach $\$ 3.7$ billion in 2016 . Figure-4 shows the wireless sensor market revenue forecast between 2003-2012 and industrial wireless sensor market in terms of percent revenue during 2009.

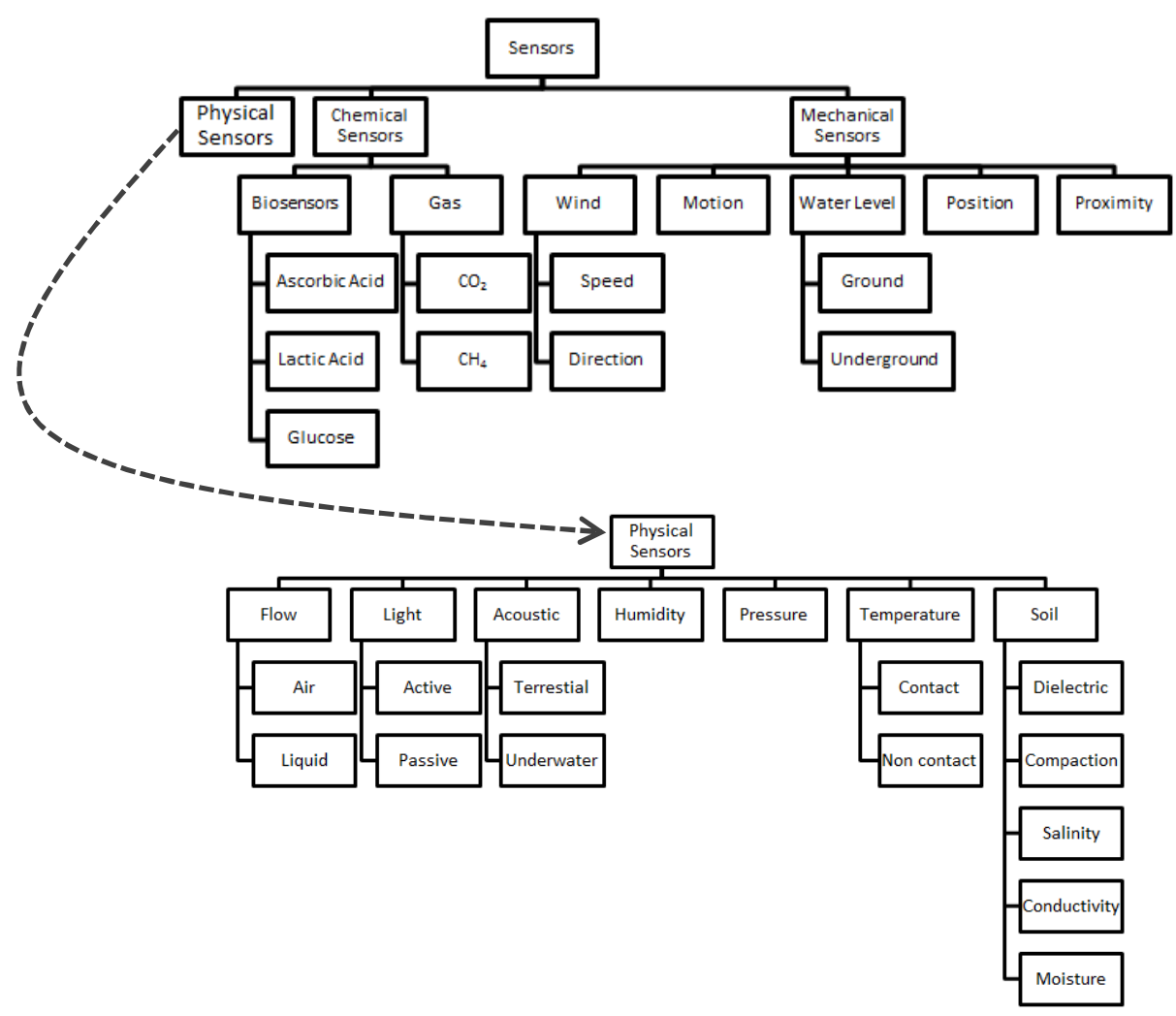

Figure 2 - A non-exhaustive hierarchy of sensor classification based on properties

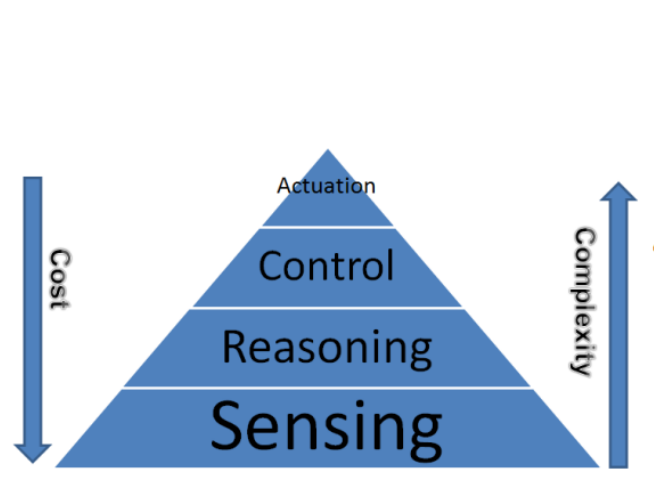

(a) Functionality of sensors

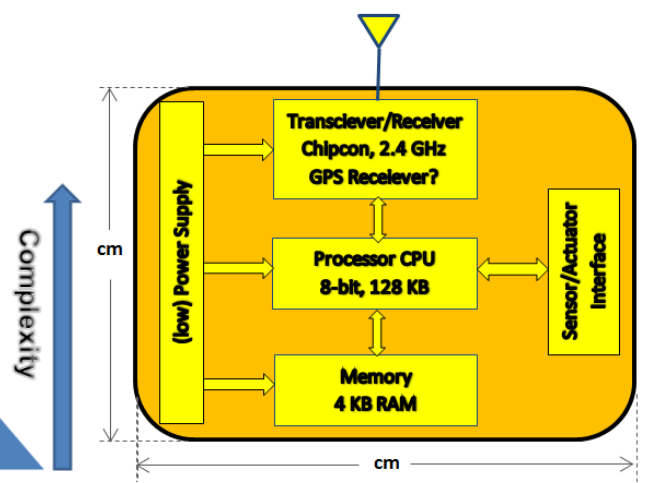

(b) Sensor architecture

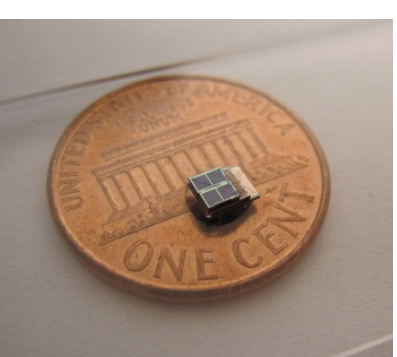

(c) Low power sensor

Figure 3 - Wireless Sensor Network Node

\section{Types of Sensor Networks}

Sensor networks allow distributed sensing capacity, real-time data visualization and analysis. Sensor networks also allow integration with adjacent networks and remote sensing data streams. Underpinning the development of networks is electronics miniaturization, availability of massive data storage, computational capacity, and the Internet. Applications involving WSNs are very diverse and involve one or a combination of various types of sensor networks. From the analysis of the WSN around the world, five types of WSNs can be identified (Meratnia et al. 2010). In this section, we will briefly discuss these WSNs with reference to smart agriculture applications discussed in sections 4 and 5 . 


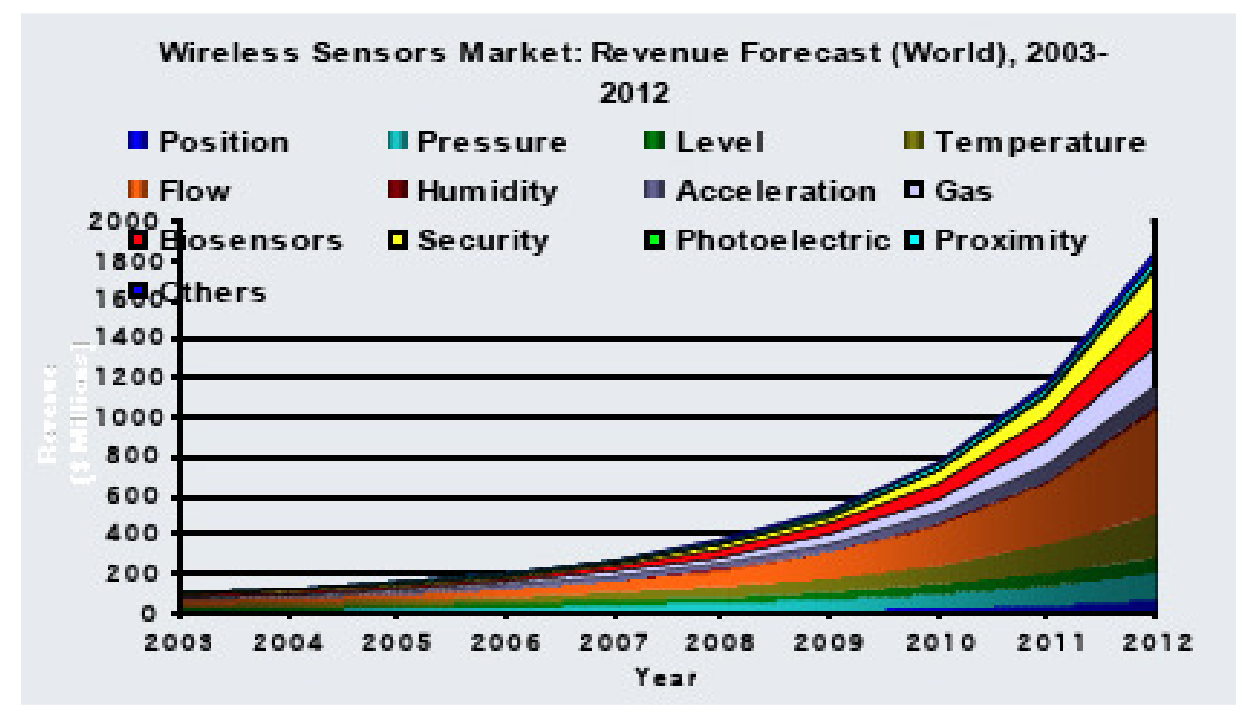

Figure 4 - Wireless sensor market actual revenue since 2002 and projected forecast for 2009-2012 (Source: Frost \& Sullivan report by Thusu, 2010)

\subsection{Environmental Sensor Network (ESN)}

Environmental sensor networks are the pioneers of wireless sensor networks. Usually, environmental sensor networks were exclusively deployed for data collection and monitoring. Environmental sensor networks are often static, nondense, large scale, and are deployed in harsh and unsupervised settings. Many are subject to harsh elements of environment that cause swift device and sensor failure. Heterogeneity of ESNs has been more with regard to different types of sensor nodes (e.g., resource-limited nodes, gateways, routers) rather than types of sensors deployed. The latest networks of this type expand their single-hop communications capability to also support multi-hop communications and likely to have more than one sink node. A recent example of ESN is monitoring of dangerous gases in manure storage facilities (Murphy, 2012), this is discussed in section-5.

\subsection{Body Sensor Network (BSN)}

Body sensor networks (BSNs) typically consists of very few wireless sensor nodes attached to a living body unified with one or more powerful personal device (e.g., smart phone). BSN usually monitor critical signs, tracking, and data collection; this being the main purpose of using these sensor networks. Normal practice has been centered on off-line analysis of collected data by experts and providing feedback mainly in the field of health and well-being (but this has changed lately). The terms Body Sensor Networks (BSN) and Body Area Network (BAN) have often been used in related applications.
Generally BAN mainly refers to the network aspect of wearable sensing applications. Conversely the BSN mainly refers to the infrastructure of implantable and wearable sensing applications. As compared to ESNs, BSNs are small scale, heterogeneous (in terms of different types of sensors) and require single-hop communication. Since various types of personal information can be collected by BSNs, both security and privacy are major concerns. Unlike ESNs, in static BSNs energy consumption is not a major issue, because dependable data processing and well-timed feedback are of higher importance, however, same is not true for mobile BSNs. A recent example of BSN is body temperature monitoring of farm workers to prevent thermal exhaustion (Matthew, 2012); this is discussed in section-5.

\subsection{Structure Sensor Network (SSN)}

Structure sensor networks consists of medium to large number of wireless nodes usually deployed in specific sites such as industrial locations or attached to buildings (e.g., office), structures (e.g., bridges) or infrastructure (e.g., rails). As compared to ESNs that are almost always deployed outdoors, SSNs may be installed both indoors and outdoors and combine several environments simultaneously, including restricted access. In terms of security, SSNs are often more security centric than ESNs and require protection mechanisms against attacks and corrosive effects of the surroundings. Due to their difficult deployment and energy efficiency, long network lifetime are very important. Similar to most conventional environmental sensor networks, SSNs are 
usually static. Structure sensor networks may be both single and multi-hop (depending on their scale) and are often heterogeneous (in terms of both type of sensors sensor nodes and functionality). As mentioned in section-3.2, monitoring of dangerous manure gases is an example of SSN.

3.4 Transport and logistics Sensor Network $(T S N)$

Lately numerous efforts have been focused towards wireless communication and networking between transportation vehicles (cars, trucks, and trains). This has resulted in development of a number of communication standards such as IEEE $802.11 \mathrm{p}$ (for vehicle to vehicle communication). Each individual vehicle can be considered to be a sensor node, which locally observes its own status while monitoring its surroundings too. The other type of TSN is based on sensors attached to food boxes loaded in the transportation vehicle, with sensors actively monitoring the transport conditions of food and reporting the measurements (unlike passive RFID). This allows reacting to changes in the transport conditions early and appropriately (Becker et al., 2009). Depending on the application, TSNs may be either in the form of a network of vehicles or a combination of vehicle networks with multiple sensors attached per vehicle. A recent example of TSN is food tagging by FoodLogiQ(www.foodlogiq.com) whereby the produce is tagged and tracked from growers to the retail outlet.

\subsection{Participatory Sensor Network (PSN) or} $M 2 M$

In its most basic form, M2M (Machine to Machine) involves devices that communicate independently i.e. without human intervention. Under M2M everyday objects are locatable, addressable, recognizable, readable, and controllable through the Internet (www.ntc.org). In fact, M2M is now synonymous with the "Internet of things" (Augmented data, www.economist.com, 2010). Current advances in mobile technology have stretched PSN functionality to the level that making and receiving phone calls are considered rather rudimentary tasks. More and more mobile phones are now supplied with sensors (e.g., GPS, accelerometer, gyroscope, camera) and different types of connectivity mediums (bluetooth, wifi, GSM, etc.). This combination makes the mobile phone and in fact people carrying them a valuable source of gathering and transmitting data. A recent example of M2M is Zebra Net (Zhang, 2008) where the Zebras were tagged with wireless sensors, and the information was used for monitoring their activities, details in section-5.3

\section{Types of Agriculture}

Agriculture is one of the most extensive activities of the world; however its character is not uniform all over the world. Various types of agriculture are practiced in the world under various socioeconomic and physical conditions called as agricultural systems.

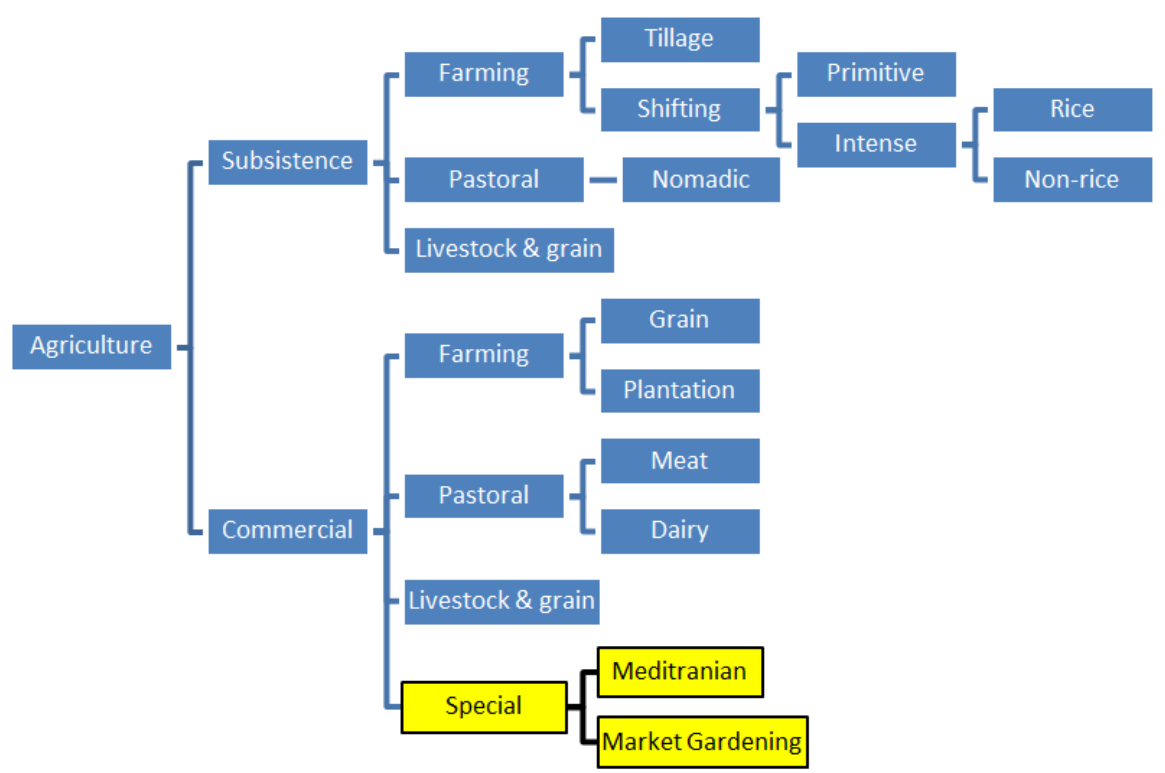

Figure 5 - Classification of Agriculture Types 
The two main types of agriculture being practiced are subsistence farming and commercial farming. Commercial farming relies on careful planning and skilled management in order to get high yield with affordable price for the customers. Commercial farming not only it pays for its own production but also earns a profit. Thus commercial farming is most suited for smart agriculture. Although subsistence farming is somewhat rare in the developed world, however, it is still an important source of food for millions in the developing world.

Whittlesey (1936) has classified agriculture based on a number of criteria, some of the major criterion being scale of farming, combinations of crop and livestock, farming intensity, ways and means of disposal of the farm produce, the level of farm automation etc. In this section, we will briefly describe the 13 major types of agriculture practiced in the world (Figure-5) and subsequently compare these types w.r.t different features of WSN i.e. i) covered area, ii) network life-time, iii) mobility, iv) density, and v) diversity.

\subsection{Nomadic Herding}

This is subsistence activity and people depend on animals for food, clothing, shelter and transport. This type of agriculture is based on raising animals on natural grasslands. This is practiced by the people of the arid and semi-arid regions and mostly in developing countries. These people are always on the move with their animals in search of water and natural pastures, thus leading a nomadic life style. The types of the animals reared differ from region to region. For example, in tropical Africa, cattle are the most important livestock, in Sahara and Asiatic deserts, sheep, goats and camel are reared and in the mountainous areas of Tibet and Andes, llamas and yak are reared.

\subsection{Livestock Ranching}

The major change came when barbed wire was invented in 1873 by Joseph Farwell Glidden, so that the movement of the cattle could be confined without wooden or stone fences. The cattle were also protected from the external elements, such as predators. Under this system of farming, the major emphasis is laid on rearing of animals but the farmers live a settled life on permanent extensive areas called as ranches. The reared animals on ranches include cattle, sheep, pigs, chickens, turkeys, quails, horses, goats, etc. and are reared mainly for wool and meat. Special breeds of animals are reared to provide maximum yield (www.lucerogeography.com). Today ranching is not an economic activity that is carried out on isolated farms; instead it has become part of the meat processing industry. This is how it differs from pastoral nomadism. This type of farming has been established on a commercial basis in developed countries where large areas or ranches are available for animal feeding, such as the low rainfall areas of South America, North America and Australia. The rearing of animals is carried out scientifically with emphasis on genetic improvement, disease control and their health care. Most of the farm activities are carried out mechanically. Virtual fence and controlled mating of bulls (Butler et al. 2004 and Lee et al. 2008, respectively) are some of the smart applications of ranching discussed in section-5.3

\subsection{Shifting Cultivation}

This is one of the oldest types of farming generally practiced by the tribes of the rainy tropical forest of Africa, Asia and Central America. This is grain centric primitive subsistence farming, using the so called "slashing and burning" technique. The land for cultivation is acquired by clearing off the forests and natural vegetation, the land is subsequently cultivated yearly till the fertility declines. The land is then abandoned and a new plot is cleared for farming. This type of farming is done manually on small fields with simple tools, with little or no use of animal or mechanized power. Currently there is a drop in this type of farming, as it is being discouraged by the government agencies due to its land spoiling nature.

\subsection{Rudimentary Sedentary Tillage}

Tillage is the preparation of the soil for agricultural activity by agitation in various ways, such as by digging, stirring, overturning, hoeing, raking etc. Tilling can be human-powered or mechanized. This is also a subsistence type of activity but it is different from the previously discussed types of agriculture, as the same piece of land is cultivated repeatedly year after year. Soil tillage has been linked with improved fertility, which is derived from the mineralization of soil nutrients as a result of soil agitation. Lately there has been a trend of zero-tillage or low-tillage.

\section{Farming}

\subsection{Intensive Subsistence Rice Dominant}

This type of intensive subsistence farming is practiced in densely populated monsoon countries of Asia such as China and India. The major emphasis is on paddy, multiple cropping and in- 
tercropping is also common in this system. Due to high input of the family labor yield per acre is high and fields are small due to dense population. Unlike primitive subsistence agriculture, animal power is used, and to maintain fertility of the soil instead of using ash of burnt vegetation, farm yard manure is used. Using sensors for noting soil moisture and weather parameters for optimum usage of water is one application of smart paddy agriculture discussed in section-5.1

\subsection{Intensive Subsistence Non-Rice Domi-} nant Farming

This is a somewhat dry climatic alternate of the rice dominant type as the amount of rainfall is not enough in these regions. Therefore, instead of growing rice, grain crops such as wheat and millets are grown. Besides the relatively dry areas of Asia, Northern Africa and the parts of Middle East, this type of non-paddy farming is usually practiced in parts of Southern Africa and also Central America.

\subsection{Commercial Plantations}

As the name implies, this is a purely commercial endeavor and practiced due to its high commercial value. This is a highly capital oriented farming with most crops being tree crops. Large areas or plantations are developed where a single crop is grown. Cultivation is done using scientific methods and professional managers and technically sound staff are employed. The major produce of this type of farming are the tropical crops such as rubber, coffee, oil palm and tea etc. This type of farming has developed in parts of Latin America, Asia and Africa where the impact of the European colonialism had been significant. The plantations were mostly developed to meet the European markets needs of tropical crops. For example, cocoa and coffee plantations were developed in West Africa by the French, while tea gardens were established in India and Sri Lanka by the British. Using field cameras combined with hyperspectral satellite imagery, to identify best time for tealeave picking (Arai, 2010) is an example of smart agriculture for tea plantations.

\subsection{Mediterranean Agriculture}

The name is derived from the Mediterranean region of Europe with representative agriculture characteristics. Both cash and subsistence crops are important for the economy of the region. Mediterranean agriculture consists of four distinct, but interrelated agricultural systems of crop production and livestock raising, which are i) grow- ing vegetables and cereals aided by the seasonal precipitation ii) cultivation of olives, figs, dates and grapes that ripen in autumn (fall) without irrigation iii) raising of widely distributed summer crops of fruits, vegetables and forage plants by irrigation and iv) raising livestock usually consisting of small animals that graze on highlands during winter and on lowlands in summer. Using wireless nodes with cameras to detect the effect of disease, virus, pest or other agents on vineyard leaves and then informing the farmer (Lloret, 2011) is an example of smart agriculture for vineyards discussed in section-5.1

\subsection{Commercial Grain Farming}

Commercial grain farming consists of large, highly mechanized operations and exists only in temperate zones. It is fairly recent, as it did not exist before mechanization (www.ca.uky.edu/agripedia). Thus this type of farming is a response to farm mechanization, and is the major activity in areas of low rainfall and low population density where extensive farming is practiced. This type of farming is concentrated around prairies, steppes and the temperate grasslands of South America and Australia. More specifically, large-scale commercial grain production is only found in a few countries such as the United States, Canada, Argentina, Australia, France and the UK. China and India are major grain producers but through subsistence agriculture (www.lucerogeography.com). Grain crops are susceptible to the whims of weather and droughts and monoculture of wheat being the general practice. Cereal grain yield sensors are well established commercially as part of smart agriculture, non-cereal grain yield monitor (Maharlouie et al. 2011) is discussed in section-5.1 as part of smart agriculture.

\subsection{Livestock and Grain Farming}

This type is commonly known as mixed farming and excluding Asia, this practice has originated in the humid areas of the middle latitudes. In this type of farming, most crops are fed to the animals instead of being directly consumed by humans. Almost all land is devoted to growing crops, but more than three fourths of the farm's income is received from the sale of animal products. Although with this type of agriculture most income occurs during the harvest season, however, livestock products can be sold throughout the year (www.lucerogeography.com). Thus the development is closely related to the market facilities, and it is typically a European type of farm- 
ing where efforts are made to get the best out of crop farming and animal rearing. New-Zealand and Britain are the example areas where this is commonly practiced. Hay tracking using RFID tags is one application of this type of smart agriculture discussed in section-5.5

\subsection{Subsistence Crop and Stock Farming}

This type of farming resembles the earlier type in terms of the crops and type of livestock, however, the difference is that almost nothing is sold off the farm. This type of farming is common in developing countries and areas of middle latitudes with lower soil fertility or areas with rough terrain. Having no surplus cash income, the farmer cannot buy expensive farm machinery nor can save the best seeds for his fields or buy breeding stock, thus is unable to get out of the vicious cycle.

\subsection{Dairy Farming}

Dairy farming has its origin in Europe from where it diffused to other parts of the world. Close vicinity to the market and a moderate climate are the two factors that are favorable for the development of this type of farming. Dairy farming is labor oriented. The cows must be taken to the milk parlor twice a day and require constant care through the year. Milk freshness is critical; therefore, farms located farther from consumers usually sell their output to those processors that make butter or cheese, or dried, evaporated, and condensed milk. Almost all the milk in Wisconsin is processed as compared to only 5 percent in Pennsylvania (www.lucerogeography.com). Countries like Sweden and Denmark have seen the maximum growth of this type of farming. Battery-less temperature monitoring of dairy cows is a smart application of dairy farming discussed in section-5.3

\subsection{Market Gardening}

It is the commercial growing of flowers, vegetables, fruits, and other plants on a scale greater than a home garden, but small enough such that most of the gardening principles are applicable. Like all commercial farm enterprises, the objective is to run the operation as a profitable business (www.agmarketing.extension.psu.edu). Market gardening is usually oriented toward local markets, however production and shipment to faraway markets is also possible. In such a case some of these products are sold fresh, but most are sold to large processors who can freeze the produce. This type of farming has been developed to take advantage of the demand for horticulture products by the population at large scale urbanized and densely populated areas of Europe. This type of farming has flourished in the vineyard cultivation areas of Swiss Lakes regions, France and northern Hungary and greenhouses have also been used for growing assorted fruits and vegetables. The farmers are willing to experiment with fertilizers, new seed varieties and other inputs to minimize costs and maximize efficiency (www.lucerogeography.com) and are thus prime candidates for smart agriculture, example and details in section-5.2.

For each type of sensor network discussed in section-3, there are a large set of hidden conditions and functionalities offered. Although it may seem difficult to clearly distinguish between different types of sensor networks with reference to different types of agriculture, however, we will do a comparison based on some important factors such as i) covered area, ii) network life-time, iii) mobility, iv) density, and v) diversity. These factors are briefly described as follows.

Network lifetime is possibly the most important metric for the assessment of sensor networks. Obviously, in a resource-constrained environment, the depletion of a limited resource must be taken into account. It could be impossible or problematic to recharge the battery in the remote location therefore, the critical requirement is to extend the network lifetime. For network coverage in WSNs, each sensor node attains a certain "view" of the environment. A sensor's coverage of the environment is limited by its accuracy and range; it can only cover a limited physical area of the environment with missing details being interpolated. High sensor density is required to assure measurement redundancy and to provide a deeper understanding of the variation of the parameter monitored, and to also monitor at the individual object level. For example in a cattle monitoring WSN, a sensor is attached to every animal, so sensor density is high. Sensor diversity is based on sensor nodes having diverse capabilities in terms of energy supply, storage space, communication, computation, reliability and other aspects. Observe that sensors, gateway, base station also result in heterogeneity of a WSN. Sensor mobility is categorized by the node additions and failures as well as physical movement of the nodes. Physical mobility is caused by the controlled movement of objects (vehicles) or "random" movement of animals with sensor nodes attached 
to them. Table-1 presents a comparison between different agriculture types using these parameters (here Homo. is Homogenous and Hetro. is Heterogeneous).

Table 1 - A comparisoin between different agriculture types w.r.t WSN parameters

\begin{tabular}{|c|c|c|c|c|c|c|c|c|c|c|c|}
\hline \multirow[t]{2}{*}{ Type of Agriculture } & \multicolumn{3}{|c|}{ Covered Area } & \multicolumn{2}{|c|}{ Life-time } & \multicolumn{2}{|c|}{ Mobility } & \multicolumn{2}{|c|}{ Density } & \multicolumn{2}{|c|}{ Diversity } \\
\hline & Large & Medium & Small & Long & Short & Mobile & Static & Low & High & Homo. & Hetro. \\
\hline 1. Nomadic Herding & & $\checkmark$ & & $\checkmark$ & & $\checkmark$ & & & $\checkmark$ & & $\checkmark$ \\
\hline 2. Livestock Ranching & & & $\checkmark$ & & $\checkmark$ & $\checkmark$ & & & $\checkmark$ & & $\checkmark$ \\
\hline 3. Shifting Cultivation & & & $\checkmark$ & & $\checkmark$ & & $\checkmark$ & $\sqrt{ }$ & & $\checkmark$ & \\
\hline 4. Rudimentary Sedentary Tillage & & $\checkmark$ & & & $\checkmark$ & & $\checkmark$ & $\checkmark$ & & $\checkmark$ & \\
\hline $\begin{array}{l}\text { 5. Intensive Subsistence Farming with Rice } \\
\text { Dominant }\end{array}$ & & & $\checkmark$ & & $\checkmark$ & & $\checkmark$ & $\checkmark$ & & $\checkmark$ & \\
\hline $\begin{array}{l}\text { 6. Intensive Subsistence Farming Without } \\
\text { Rice Dominant }\end{array}$ & & $\checkmark$ & & & $\checkmark$ & & $\checkmark$ & $\checkmark$ & & $\checkmark$ & \\
\hline 7. Commercial Plantations & & $\checkmark$ & & & $\checkmark$ & & $\checkmark$ & & $\checkmark$ & & $\checkmark$ \\
\hline 8. Mediterranean Agriculture & & $\checkmark$ & & $\sqrt{ }$ & & $\checkmark$ & $\checkmark$ & $\sqrt{2}$ & $\checkmark$ & $\checkmark$ & $\checkmark$ \\
\hline 9. Commercial Grain Farming & $\checkmark$ & & & & $\checkmark$ & & $\checkmark$ & $\checkmark$ & & $\checkmark$ & \\
\hline 10. Livestock and Grain Farming & 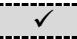 & & $\checkmark$ & & $\checkmark$ & $\checkmark$ & $\sqrt{ }$ & $\sqrt{2}$ & $\checkmark$ & $\checkmark$ & $\checkmark$ \\
\hline 11. Subsistence Crop and Stock Farming & & & $\checkmark$ & $\checkmark$ & & $\checkmark$ & & & $\checkmark$ & & $\checkmark$ \\
\hline 12. Dairy Farming & & & $\checkmark$ & $\checkmark$ & & $\checkmark$ & & & $\checkmark$ & & $\checkmark$ \\
\hline 13. Specialized Horticulture & & & $\checkmark$ & & $\checkmark$ & & $\checkmark$ & & $\checkmark$ & & $\sqrt{ }$ \\
\hline
\end{tabular}

From Table-1 it can be observed that Mediterranean Agriculture is rich in sensor applications. For example, considering vineyard the sensor density is high though sensors are static, con- sidering cattle, the sensor density is till high but the sensors are mobile. Based on the type of crops and area coverage there is a variety in the nature of sensors.

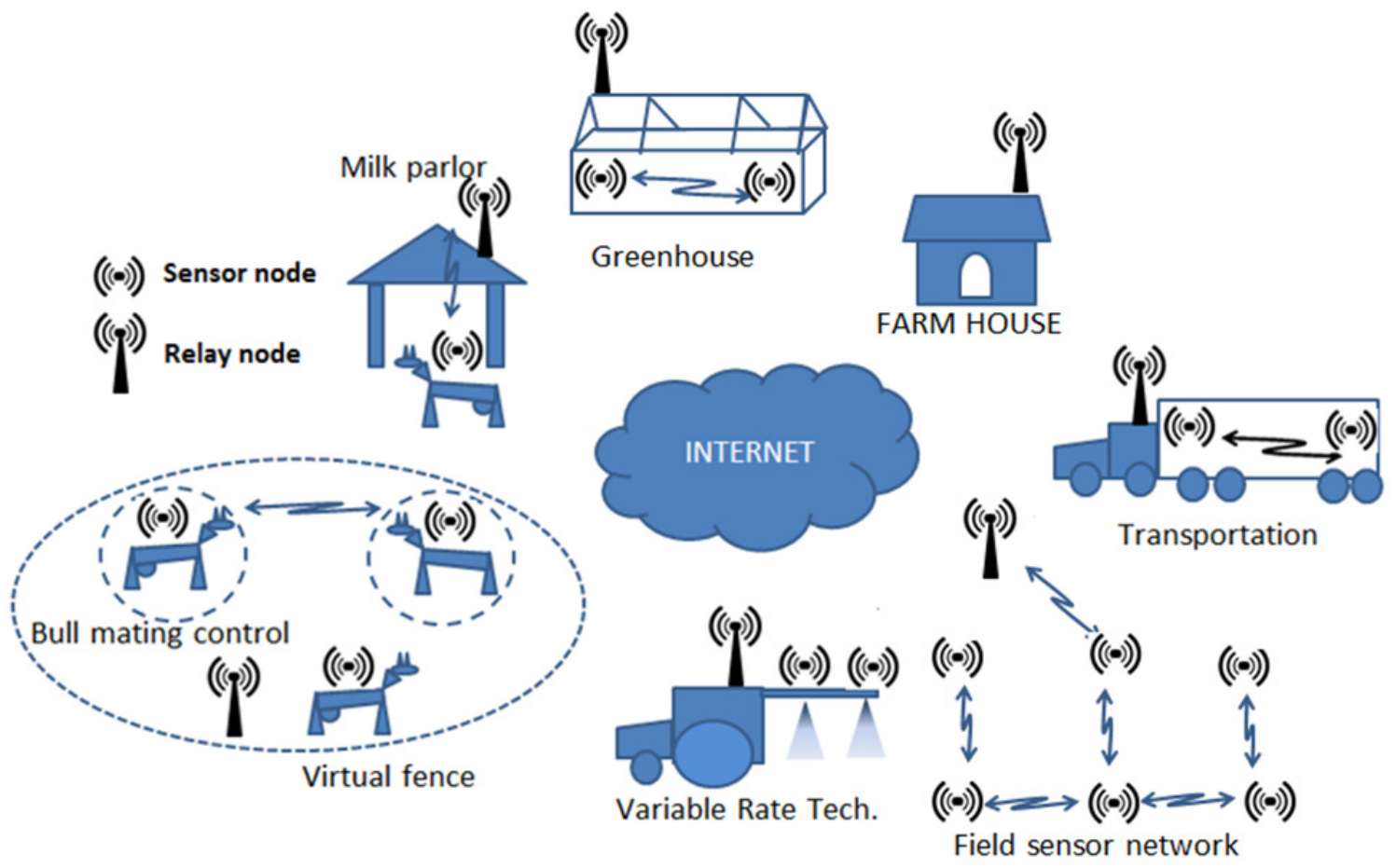

Figure 6 - Wireless sensor networks in smart Mediterranean agriculture

\section{Applications of Sensor Networks in Mediterranean Agriculture}

In this section we will discuss some recent applications of sensor networks suitable for Mediterranean agriculture. The applications considered consist of some of the comparatively more established ones such as Precision Farming and Greenhouse, along with some of the more upcoming applications such as cattle monitoring and food quality monitoring/tagging to name a few. 
Figure-6 shows a smart Mediterranean farm with multiple nested levels of WSN consisting of the relay nodes and the sensor nodes. Note that the relay nodes are used to expand the WSN coverage throughout the smart farm. The first level consists of the "wireless cloud" that connects broadband Internet to the farm along with connectivity with far flung TSN. The second level of network is the farm-wide WSN with both monitoring and actuation nodes. The third level of nested network is a WSN at the field level where it is used to monitor the field parameters. The fourth nested level is the PSN or M2M between the cattle being reared on the farm/ranch. The fifth level is the hovering UAV (Unmanned Aerial Vehicle) which is a mobile wireless node; UAV is used to collect data from the sensors while it passes over them and subsequently transmits it to the farmhouse. The sixth and possibly the last level is the agriculture worker net without a relay node.

\subsection{Precision Farming}

Although Precision Farming (PF) started as a technology-driven development, it is not just another name for yield mapping and variable rate technology (VRT) for managing spatial variability within a field. Instead, PF integrates analysis and synthesis for crop production with the goal to reduce decision uncertainty. This is achieved by using a data driven approach, through better understanding and management of natural variation. In PF expertise from many disciplines is utilized, including information technology, telecommunication engineering and GIS to name a few. Thus the coverage of PF is diverse which includes, but is not limited to crop growth monitoring, global positioning systems, remote sensing, soil fertility, electronic equipment, global information systems, computer models, decision support systems, variable-rate technology, yield mapping and accurate record-keeping. PF potentially leads us to "Push Button Agriculture". Discussion of all applications of PF is beyond the scope of the paper; therefore, in this section, we will only discuss a few recent applications.

A disease, a deficiency, pest, or other harmful agents can cause morphological or physiological changes in the vine plant. Therefore, the symptoms can be visually spotted because of the change of the green color and/or appearance of some color stains on the leaf. The proposed wireless sensor network by Lloret et al. (2011) is based on a collection of nodes capable of capturing images, processing them locally and generating corresponding responses based on its decision i.e. whether or not a bad (infected/damaged) leaf was found. This results in a distributed system, where each node makes its own decision, thus avoiding network contention and overwhelming the central server. The proposed system however was not able to distinguish between deficiency, pest, disease or other harmful agents. Some of the problems faced were i) misinterpretation of bad leaves with the ground because of similarity of color and ii) variation in size of the same leaves based on images taken at different distances.

\subsection{Greenhouse}

Historically farmers have experienced huge financial losses because of wrong weather predictions and incorrect irrigation timings (Chaudhary et al. 2011). As per FAO (2011) Report, climate change is expected to further aggravate the existing challenges that agriculture faces. In this context, with the evolution in wireless sensor technologies and miniaturized sensor devices, it is possible to use WSN for automatic environment monitoring and controlling the parameters in a confined environment i.e. a greenhouse. Greenhouses have been in use for decades, but it is not until recently that there has been an increase mainly due to availability of diverse range of sensors and actuators with rich sensor applications. Some of the climatic control variables affecting a greenhouse are shown in Figure-7 (Pawlawski et al. 2011).

The greenhouses in the warmer Mediterranean region are characterized by some fundamental differences as compared to their North Western European counterparts in terms of the temporal distribution and "quantity" of natural resources, such as radiant energy. These climatic differences have resulted in the development of a greenhouse system based on simple structures and low-cost climatic control systems having some intrinsic shortcomings. Some of those shortcomings being poor humidity and ventilation control along with reduced light transmission through the plastic coverage. Smart WSN applications are therefore needed to control actuators like pump, valve, carton slider and fans etc. (Chaudhary et al. 2011). The Greenhouse control can be achieved through WSN via Ethernet connected to the central PC of a remote network. Bluetooth technology can be used for some applications to collect environmental data from a sensor network and transmit to a central control system. This type of remote control technology significantly improves productivity and reduces the labor cost. 


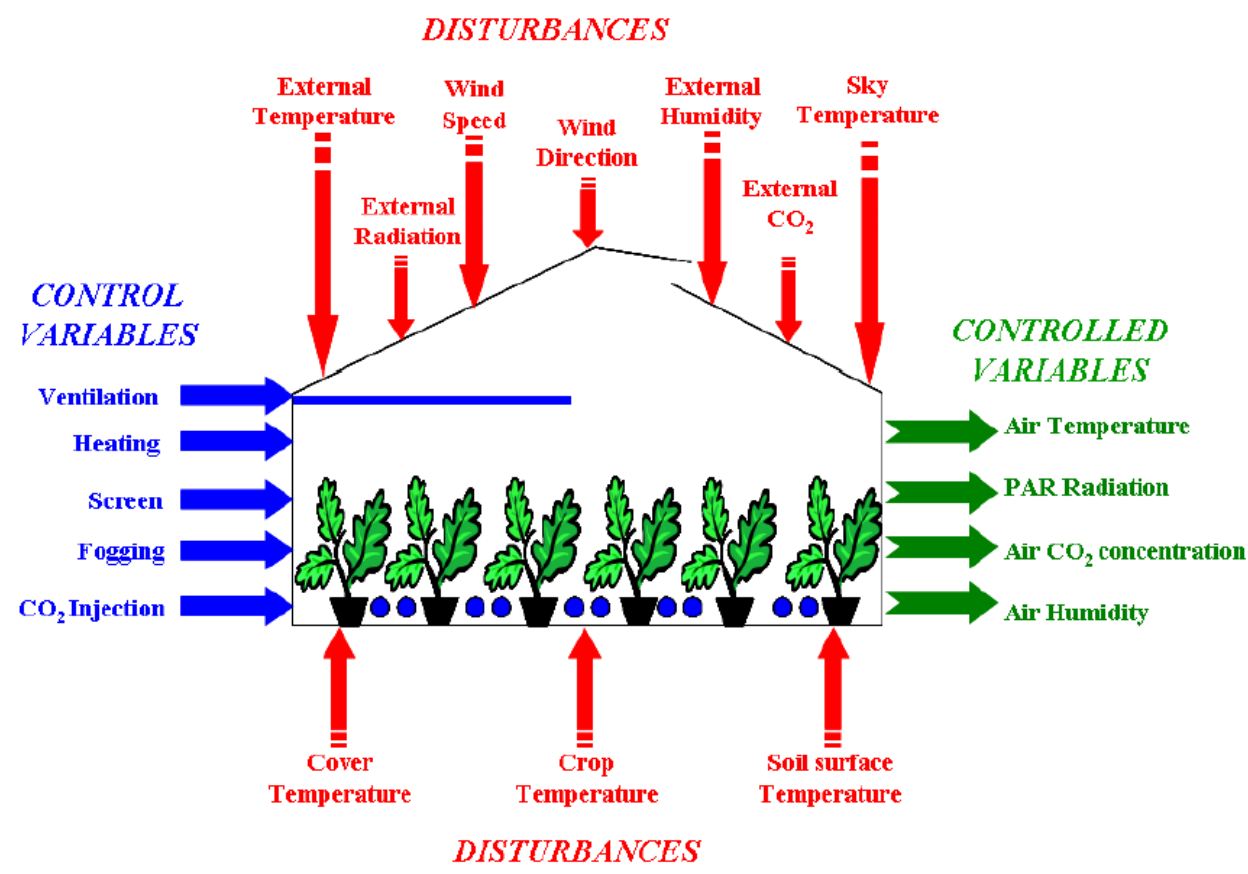

Figure 7 - Climatic control variables in a greenhouse

Crop growth in a greenhouse is effected by dew condensation on the leaf surface of crops that can stimulate diseases caused by bacteria and fungus. Park et al. (2011) have presented a WSNbased automatic monitoring system solution that prevents dew condensation in a greenhouse environment. The system consisted of sensor nodes for collecting data, base nodes for processing collected data, relay nodes for driving devices for adjusting the environment inside the greenhouse and an environment server for data storage and processing. Barenbrug formula was used for calculating the dew point on the leaves; the system was able to prevent dew condensation phenomena on the crop's surface for prevention of diseases.

Lee et al. (2011) have discussed a 'Paprika Green House System' (PGHS) that consists of a network of sensors measuring temperature, humidity, illuminance and other relevant parameters. The PGHS also controls ventilators, humidifiers, lightings and video-processing through a GUI application, this is achieved by analyzing the measured data. The system also maintains the best environment in the cultivation facility by using biometric data and creates optimum conditions at paprika root zone. The system optimizes the management of production elements and reduces energy, fertilizer and water loss resulting in reduction of production costs. To overcome the issue of separate conversion/control module for each sensor characteristic, an integrated sensor module was developed. This module integrates various sensors required for cultivation of the crop into a single node. LED used in PGHS prevented vermin and adjusted the growth rate and crop coloring so that shipping timings could be adjusted. The "greenhouse environment integrated management system" enabled the monitoring of PGHS in real-time through Internet.

\subsection{Cattle monitoring}

Usually a farmer relies on a combination of visual observation, experience and instinct while making management decisions about the herd, but these data intensive decisions made on intuition are likely to be far from optimal and also labor intensive. Agricultural community has a continued interest of using sensor networks to monitor and control livestock. The use of sensor networks with livestock allows farmers to monitor and control the herd even when the farmer is far from the field. This monitoring and control can help detect illness-related behavior, enhance land management, and develop animal behavior models. Mobile sensor network nodes can thus be used to influence and monitor animals' positions in the pastures and grasslands (Wark et al. 2007).

One of the first major applications of WSN for animal monitoring was tracking of zebras reported by Zhang et al. (2008) as part of the ZebraNet project. ZebraNet used the zebra's GPS position data that was taken every few minutes. 
This data was subsequently hopped in a peer-topeer fashion (as in PSN) to other zebras when they came in range. Subject to the amount of storage capacity of each device, a user could then download historical position data of multiple zebras by approaching a single zebra. Researchers have since proposed advanced systems for ad-hoc routing of data through large networks of mobile cattle nodes (Radenkovic and Wietrzyk, 2006).

Both Butler et al. (2004) and Lee et al. (2008) have used similar stimuli but achieved different goals using different algorithms. Butler et al. (2004) developed a "moving virtual fence" algorithm for herding cows. Each animal in the herd was given a smart collar consisting of a sound amplifier, a PDA, a GPS and a radio unit (WLAN). The animal's location was determined using the GPS and confirmed through a measurement of vicinity of the cow relative to the fence boundary. When approaching the perimeter, the animal was given an auditory stimuli, which pushed the animal away from the fence. Lee et al. (2008) have examined the potential of controlling the bulls during mating using mild electric shocks delivered through radio controlled collars. Cows were assigned to the bulls; the nonassigned bull received a mild electric shock on approaching either the unassigned cow or another bull. Non-assigned bulls were sometimes observed avoiding the cow despite a change in its location. This suggests that the bull associated the electric shock with the cow and not with the location in which they received the electric shock.

The US dairy industry is merging rapidly. Today fewer dairy operations are family operated, therefore in larger, more mechanized dairies the attention and care given to each dairy cow has consequently reduced. As a result mortality rates and production loss from sickness are on the rise in these large dairy operations (www.phaseivengr.com). A possible solution to these problems is the Dairy Monitoring System (www.phaseivengr.com) that consists of i) a bolus, equipped with a passive RFID chip, an embedded temperature sensor and ii) a reader for collecting temperature data from the bolus. Once the bolus is orally administered, it settles into one of cow's stomachs. The density of the bolus prevents it from exiting the digestive system. As the dairy cow passes between two panels fixed at the entry to the milking parlor, a small electrical charge is induced inside the bolus by a magnetic field. This charge is sufficient to energize the transmitter. The bolus responds to this query signal by transmitting its unique ID and temperature on a coded radio-frequency. As the dairy cow enters the milking parlor two to three times per day, the system automatically monitors each cow's temperature and ID. This allows the dairy operator to receive a 24 to 48 hour advance notice on any outbreaks or illnesses before visible signs appear, thus reducing treatment costs and mortality rates.

\subsection{Food quality monitoring and tagging}

The selection of a particular foodstuff by a consumer is influenced by many sensory observations; one of them being taste. Taste is affected by different factors including sweetness, saltiness, bitterness and acidity; these being the most important factors. Texture is another key sensory observation and is influenced by many factors including moisture content, carbohydrate and protein levels (Szczesniak, 2006) in the foodstuff. Other important sensory observations include the shape, scent, and color of the foodstuff. Therefore, rapid, portable and accurate sensors are required for automatic assessment of food quality and physiological state. Due to their numerous attributes, biosensors potentially offer a fast, accurate, relatively cheap, portable, stable, and user-friendly mechanism for on spot monitoring of fruit maturity and quality (Rana et al. 2010).

As per Kriz et al. (2002), monitoring of fruit quality is one of the major worries within the food industry. There is a growing need to develop analytical devices, which can provide quality monitoring for the entire food processing operation i.e. starting from the materials to the end products (Whitaker, 1993). Sensors incorporated into food packages can potentially benefit consumers, by guaranteeing freshness and quality, and at the same time allowing retail industry to more effectively manage food stocks and product genuineness.

Meats and fish are prone to rapid growth of bacteria. Bacterial growth on meats and fish typically results in release of nitrogen and sulfur containing compound, accompanied by strong and foul smell. Smits et al. (2012) used smart radiofrequency labels with sensors enabling measurement of temperature, humidity and the presence of volatile amine compounds. The labels were made by means of high quality screen printing on low cost foils using lamination technologies. This was combined with pick and place technology. 
For data processing, commercially available cost effective MSP430G microprocessors from Texas Instruments were used.

Hay harvesting is a precise process with a significant bearing on the success of a farm's feeding program. Hay is not only used for the nourishment of livestock, but the harvested hay to be of top quality so that it fetches a good price. Hay is at its best through a particular week-long period during its maturation. Beyond this point, it becomes coarse and dry losing most of its nutritional value. Tracking the moisture levels in hay bales is one of the important activities of the entire hay baling process. Any bale harvested below $12 \%$ moisture content has experienced field losses, resulting in low feeding value and yield losses. A bale with moisture content above $20 \%$ is at risk of spontaneous combustion due to increased heat levels during the respiration process (www.thingmagic.com).

In the smart solution (www.thingmagic.com) a thin RFID tag is attached to one of the coils holding the hay together. On this small tag, all essential data is stored. With an RFID reader, anyone can check the field of origin of the hay with exact location of harvest, harvest date, average and high moisture levels, temperature, weight, amount of preservatives used, and a unique ID number. With this useful information, farmers can dispense hay to their livestock and ensure its consistent quality. The remaining hay can be sold for a good price since the buyer is aware of the exact nutritional value of each bale. Bales with too much moisture are removed from a stack to avoid contamination of the remaining bales. These bales are subsequently used to feed cows or put to other uses where high quality is not a priority.

\section{Challenges}

Adoption, utility and applications of WSNs in agriculture is not without multitude of challenges and the requirement of addressing difficult research problems. Some of those generic challenges are mentioned in this section.

\subsection{Cost per unit}

A major obstacle to wider embracing of wireless sensor networks is the cost of motes. At $\$ 99$ to \$300 apiece (Madan and Reddy, 2012), motes are currently too costly for many of the applications its inventors envisioned, such as extensive use in agriculture. One idea calls for wireless sensors that you "peel, stick and forget" (Smits et al. 2012). The radio frequency identifi- cation (RFID) tag industry possibly has reached a cost as low as about $\$ 0.20$ per tag and seeks to reach in a decade, the price of $\$ 0.05$ per tag for inventory tracking purposes (Homs et al., 2004).

\subsection{Battery Life}

Wireless sensors run on batteries--which can create maintenance botheration if users are expected to replace them regularly and that too for hundreds and thousands of sensors. Without energy, a sensor is basically useless and cannot contribute to the usefulness of the network as a whole. However, there are upcoming WSN applications where sensors are required to operate for much longer periods (like years or even decades) after being deployed.

\subsection{Data Fusion and Quality}

Location (the sensor node or base station) of data processing is another critical issue, because data processing at the sensor node consumes energy and is limited by the device capacity, however, this saves transmission energy and network congestion. The correct trade-offs on processing location seem to be system dependent. At the same time data gathering has its own data quality issues, some of which are i) missing data ii) missing observations and iii) variations in observations.

\subsection{Signal attenuation}

Radio waves are attenuated while transmitted from the sender to the receiver. The degree of attenuation is dependent on the medium between the receiver and the transmitter. According to Becker et al (2009), in case of food, major attenuation occurs because of water, as water constitutes main part of the food having propagation attenuation worse than air. According to Gabriely et al. (1996) attenuation is also caused by the animal body, which is highest for the fat and lowest for the blood. Consider the case of using wireless sensors for monitoring cattle health, since the cattle are generally fed in herd, this results in massive increase in the surface area, which seriously affects the radio signals. The sensor communication system is required to minimizing the impact of radio attenuation through food and animal body.

\subsection{Authenticity and Security}

Severe environmental operating conditions, hazards of physical compromise and unpredictable data transfer rates are some of the challenges for WSN. WSN are usually deployed in an unattended environment, thus the sensors could be compromised for malicious reasons, such as in- 
tentional falsification of sensor data ( $\mathrm{Yu}$ et al., 2010). In such a case, garbage in would obviously result in garbage out. The traditional solutions of data authenticity, confidentiality are there, however due to the resource constraints of the sensors, the existing protocols cannot be used for WSN. There are other constraints and obstacles too that need be resolved while designing a security protocol for WSNs. Some of these constraints being limited memory, storage and processing capabilities; these limitations differentiate WSN security architecture design requirements from traditional network design requirements.

6.6 Operating Systems

TinyOS (www.tinyos.net) is event-driven operating system which is assumed to perform better under constrained environments. Yet such OS lack some system utilities resulting in imposition of their own constraints. Nevertheless, thread-driven systems such as MOS (Bhatti et al., 2005) provide high concurrency with preemption, allowing their use in real-time applications. Research has shown the ability of thread-driven systems to outperform event-driven systems. Yet, in some cases such as high system load, the threaddriven approach usually consumes more energy. Designers therefore have to prioritize or establish a trade-off between energy consumption and high concurrency.

\section{Future Directions}

\subsection{Increasing Battery Life to Decades}

The biggest problem faced by WSN is energy. When a sensor is drained of energy, it can no longer accomplish its role unless the source of energy is replenished. Therefore, it is normally accepted that a wireless sensor dies when its battery runs out. Even when not in use, portable energy sources like batteries will experience current leakages that ultimately drain the resource; furthermore, any defects in the packaging due to long term wear and tear can result in environmental issues (Seah et al., 2009. Therefore, it is urgently needed to increase the battery life or decrease its discharge rate. One possible solution being pursued is energy harvesting. "Energy harvesters" are small devices which take ambient energy and convert that into electrical energy to power the wireless sensor. The aim is 20 year lifetime or more. Photo-voltaics are most commonly used, but there is work underway on other types of energy harvesters too, such as piezoelectrics (harvesting energy from vibrations), electrodynamics (similar to bicycle dynamo), thermo-electrics (harvesting energy from a heat gradient) and more (Raghi et al. 2011). However, these are not the major sources for harvesting energy in a typical agriculture environment.

\subsection{Reducing Amount of Data Transmitted}

With hundreds and thousands of sensors deployed, collecting and transmitting data several times a day could quickly overwhelm a system. Therefore, ways and means need to be developed in order to avoid getting drowned in data while starving for information. One way of managing the flood of data is to work on the most relevant data. For example, the traditional humidity sensors utilized in agriculture have a downside that they acquire a large amount of data which is to be processed or transmitted. Whereas the intelligent sensor by Shinghal et al. (2011) uses a traditional sensor and an embedded processor that processes the acquired data using algorithms to reduce the amount of data collected. Subsequently only relevant information is extracted and then presented in a format minimizing post-processing latency. Thus the intelligent humidity sensor reduces the amount of data processed by $50 \%$ (depending upon humidity changes) and thereby reduces the power consumption. Similar work is needed for other sensors too.

\subsection{Developing Distributed Algorithms}

A WSN is a form of distributed system, where individual sensor nodes cooperate to ensure the network as a whole meets specific application requirements. The a-priori knowledge of a node is only about its own state. In order to know about other nodes in the network, a node has to talk to its neighbors. According to Laube and Duckham (2009), by collaboration of the nodes, global operations such as (multi-hop) routing or global knowledge discovery can be achieved. Since these activities are distributed among the nodes, we need distributed algorithms (Peleg, 1987). Distributed algorithms raise many interesting research questions (Wattenhofer, 2006). Some of the questions needing exploration are: What can be achieved in a distributed fashion, and what not? How effective is a distributed algorithm compared to a corresponding global algorithm? For mobile sensors, such as used in cattle net, when the cattle move apart, there are additional challenges of overall degradation of signal quality and oscillation of the communication throughput. These critical limitations need to be 
considered while developing distributed algorithms for mobile sensors for cattle.

\section{Conclusions}

Wireless Sensor Networks (WSN) is an application rich domain with more applications on the horizon. In view of the challenges of agriculture such as data collection, monitoring, actuation, large area and harsh environment make WSN a platform of choice. Because of the diver- sity of the domain of agriculture and WSN, we have endeavored to cover some of the recent applications of WSN and classify sensors and their applications for 13 traditional types of agriculture. Based on this analysis we identify the type of agriculture most suited for WSN i.e. Mediterranean Agriculture and presented recent applications of WSNs suitable for Mediterranean Agriculture.

\section{REFERENCES}

A. Bouchama and J. P. Knochel. 2002. Heat stroke. New England J. Medicine 346, 1978.

Animal Agriculture in the World. 2009. Agripedia. University of Kentucky's College of Agriculture http://www.ca.uky.edu/agripedia/agmania IIAS/ASC106/TYPEGRAI.asp

Anonymous, Animal Health: Automatic ID and Cow Temperature Monitoring http://www.phaseivengr.com/p4main/Solu tions/WirelessSensingSolutionsInDepth/A nimalHealthandIdentification.aspx

Anonymous, www.lucerogeography.com

Argaud, L., Ferry, T., Le, Q. H., Marfisi, A., Ciorba, D., Achache, P. Ducluzeau, and D. Robert. 2007. Short-and long-term outcomes of heatstroke following the 2003 heat wave in Lyon, France. Archives of internal medicine, 167(20), pp. 21772183.

Augmented Data. 2010. Economist. http://www.economist.com/node/1738839 $\underline{2}$

Becker, M., Yuan, S., Jedermann, R., TimmGiel, A., Lang, W., \& Görg, C. 2009. Challenges of applying wireless sensor networks in logistics. Proc. CEWIT 2009. Wireless and IT Driving Healthcare, Energy and Infrastructure Transformation.

Bruinsma, J. 2009. The resource outlook to 2050: By how much do land, water and crop yields need to increase by 2050 ? Paper presented at the FAO Expert Meeting, June 24-26, 2009, Rome, on "How to Feed the World in 2050". Rome: Food and Agriculture Organization of the United Nations, Economic and Social Development Department.

Burney, J. A., Davis, S. J., \& Lobell, D. B. 2010. Greenhouse gas mitigation by agri- cultural intensification. Proceedings of the national Academy of Sciences, 107(26), pp. 12052-12057.

Butler, Z., Corke, P., Peterson, R., \& Rus, D. 2004. Virtual fences for controlling cows. In Robotics and Automation, 2004. Proceedings. ICRA'04. 2004 IEEE International Conference on (Vol. 5, pp. 44294436). IEEE.

By the Editors of Technology Review. 2003. 10 Emerging Technologies that Will Change the World. Technology Review Magazine

(MIT) http://www2.technologyreview.com/featur ed-story/401775/10-emergingtechnologies-that-will-change-the/2/

C. Park and P. H. Chou. 2006. Eco: Ultrawearable and expandable wireless sensor platform. In International Workshop on Wearable and Implantable Body Sensor Networks (BSN'06), pp. 162-165.

Chaudhary, D. D., Nayse, S. P., \& Waghmare, L. M. 2011. Application of wireless sensor networks for greenhouse parameter control in precision agriculture. International Journal of Wireless \& Mobile Networks (IJWMN) Vol, 3(1), pp. 140-149.

Chien, T. V., Chan, H. N., \& Huu, T. N. 2011. A Comparative Study on Hardware Platforms for Wireless Sensor Networks. International Journal on Advanced Science, Engineering and Information Technology, 2(1), pp. 70-74.

FAO Report. 2011. Strengthening Capacity for Climate Change Adaptation in Agriculture: Experience and Lessons from Lesotho.

Fischer, G., Shah, M., \& Harrij "van" Velthuizen. 2002. Climate change and agricultural vulnerability. IIASA, International Institute for Applied Systems Analysis. 
Gabriel, S., Lau, R. W., \& Gabriel, C. 1999. The dielectric properties of biological tissues: III. Parametric models for the dielectric spectrum of tissues. Physics in medicine and biology, 41(11), 2271.

Homs, C., Metcalfe, D., \& Takahashi, S. 2004. Exposing the myth of the 5-cent RFID tag. Forrester Research, Inc., Cambridge, MA.

Ji-woong Lee, J. W. L., Changsun Shin, C. S., \& Hyun Yoe, H. Y. 2010. An Implementation of Paprika Green house System Using Wireless Sensor Networks. International Journal of Smart Home, 4(3), pp. 57-68.

Ken Lynch. 2010. Improving Farming with RFID. http://rfid.thingmagic.com/rfidblog/bid/35678/Improving-Farming-withRFID

Kriz, K., Kraft, L., Krook, M., \& Kriz, D. 2002. Amperometric determination of LLactate based on entrapment of lactate oxidase on a transducer surface with a semi-permeable membrane using a sire technology based biosensor. Application: Tomato paste and baby food. Journal of agricultural and food chemistry, 50(12), pp. 3419-3424.

Lee, C., Prayaga, K. C., Fisher, A. D., \& Henshall, J. M. 2008. Behavioral aspects of electronic bull separation and mate allocation in multiple-sire mating paddocks. Journal of animal science, 86(7), pp. 1690-1696.

Lloret, J., Bosch, I., Sendra, S., \& Serrano, A. 2011. A wireless sensor network for vineyard monitoring that uses image processing. Sensors, 11(6), pp. 61656196.

M. Radenkovic and B. Wietrzyk. 2006. Wireless Mobile Ad-Hoc Sensor Networks for Very Large Scale Cattle Monitoring. Proc. 6th Int'l Workshop Applications and Services in Wireless Networks (ASWN 06), pp. 47-58.

Mark Tercek. 2012. Feeding the World Through Smarter Agriculture http://www.huffingtonpost.com/marktercek/world-hunger_b_1459961.html

Meratnia, N., Van der Zwaag, B. J., Van Dijk, H. W., Bijwaard, D. J., \& Havinga, P. J. 2010. Sensor networks in the low lands. Sensors, 10(9), pp. 8504-8525.
Miskowicz, M. (2005, September). Sampling of signals in energy domain. In Emerging Technologies and Factory Automation, 2005. ETFA 2005. 10th IEEE Conference on (Vol. 1, pp. 4-pp). IEEE.

Myers, J. R., \& Hendricks, K. J. 2001. Injuries among youth on farms in the United States, 1998. Department of Health and Human Services, Centers for Disease Control and Prevention, National Institute for Occupational Safety and Health.

Park, D. H., \& Park, J. W. 2011. Wireless sensor network-based greenhouse environment monitoring and automatic control system for dew condensation prevention. Sensors, 11(4), pp. 3640-3651.

Patrick Laube and Matt Duckham. 2009. Decentralized spatial data mining for geosensor networks, In Miller, H.J., Han, J., Eds., Geographic Data Mining and Knowledge Discovery, 2nd, CRC Press, London, pp. 409-430

Pawlowski, A., Guzman, J. L., Rodríguez, F., Berenguel, M., Sánchez, J., \& Dormido, S. 2010. Study of event-based sampling techniques and their influence on greenhouse climate control with Wireless Sensors Network. Factory Automation, Javier Silvestre-Blanes (Ed.), ISBN: 978-953307-024-7,

InTech, http://www.intechopen.com.

Peleg, D. 1987. Distributed computing: a locality-sensitive approach (Vol. 5). Society for Industrial Mathematics.

Raghu Das, "Wireless Sensor Networks: The Challenges and Opportunities", http://www.mpdigest.com/issue/Articles/2 011/sept/idtech/Default.asp

Rajender Thusu. 2010. Wireless Sensor Use Is Expanding in Industrial Applications. http://www.sensorsmag.com/networkingcommunications/wireless-sensor/wirelesssensor-use-is-expanding-industrialapplications-7212

Rana, J. S., Jindal, J., Beniwal, V., \& Chhokar, V. 2010. Utility biosensors for applications in agriculture - A Review. Journal of American Science, 6(9), pp. 353-375.

S. Bhatti, J. Carlson, H. Dai, J. Deng, J. Rose, A. Sheth. B. Shucker, C. Gruenwald, A. Torgerson, and R. Han. 2005. MOS: An Embedded Multithreaded Operating System for Wireless Micro Sensor Platforms, 
ACM Kluwer Mobile Networks and Applications Journal, Special Issue on Wireless Sensor Networks, Kluer Academic Publishers, Hingham, USA, pp. 563-579.

Seah, W. K., Eu, Z. A., \& Tan, H. P. 2009. Wireless sensor networks powered by ambient energy harvesting (WSN-HEAP)Survey and challenges. In Wireless Communication, Vehicular Technology, Information Theory and Aerospace \& Electronic Systems Technology, 2009. Wireless VITAE 2009. 1st International Conference on (pp. 1-5). IEEE.

Shinghal, K., Noor, A., Srivastava, N., \& Singh, R. 2011. Intelligent Humidity Sensor For-Wireless Sensor Network Agricultural Application. International Journal of Wireless \& Mobile Networks (IJWMN) Vol, 3, pp. 118-128.

Smits, E., Schram, J., Nagelkerke, M., Kusters, R., van Heck, G., van Acht, V. \& Gerlinck, G. 2012. 4.5. 2 Development of printed RFID sensor tags for smart food packaging. Tagungsband, pp. 403-406.

Stern, N. H. 2006. Stern Review: The economics of climate change (Vol. 30). London: HM treasury.

Szczesniak, A. S. 2006. Classification of Textural Characteristics. Journal of Food Science, 28(4), pp. 385-389.

The 5-cent RFID Tag. 2004. RFiD Journal 1(1). pp. 30-34

Vellidis, G., V. Garrick, S. Pocknee, C. Perry, C. Kvien, M. Tucker. 2007. How wireless will change agriculture. In: Stafford, J.V. (Ed.), Precision Agriculture '07 - Proceedings of the Sixth European Conference on Precision Agriculture (6ECPA), Skiathos, Greece, pp. 57-67. Invited Keynote Presentation.

Vini Madan and SRN Reddy. 2012. Review of Wireless Sensor Mote Platforms. VSRDIJEECE, Vol. 2 (2), pp. 50-55

W3C Semantic Sensor Network Incubator Group http://www.w3.org/2005/Incubator/ssn/wi ki/Agriculture_Meteorology_Sensor_Net work
Wark, T., Corke, P., Sikka, P., Klingbeil, L., Guo, Y., Crossman, Phil Valencia, Dave Swain, Greg Bishop-Hurley. 2007. Transforming agriculture through pervasive wireless sensor networks. Pervasive Computing, IEEE, 6(2), pp. 50-57.

Wattenhofer, R. 2006. Sensor networks: distributed algorithms reloaded-or revolutions?. Structural Information and Communication Complexity, 24-28.

Whitaker, J. R. 1993. The need for biosensors in the food industry and food research. FOOD SCIENCE AND TECHNOLOGYNEW YORK-MARCEL DEKKER-, pp. 13-30.

White, R.M. 1987. A Sensor Classification Scheme, IEEE Transactions on Ultrasonics, Ferroelectrics and Frequency Control, Volume: 34, Issue: 2, pp. 124-126

Whittlesey, D. 1936. Major agricultural regions of the earth. Annals of the Association of American Geographers, 26(4), 199-240.

Wireless Sensor-1. 2012. University of Michigan.

http://www.engin.umich.edu/newscenter/f eature-story-photos-repository/wirelesssensor-1

Yu, C. M., Chen, C. Y., Lu, C. S., Kuo, S. Y., \& Chao, H. C. 2010. Acquiring authentic data in unattended wireless sensor networks. Sensors, 10(4), pp. 2770-2792.

Zhang, P., Sadler, C. M., Lyon, S. A., \& Martonosi, M. 2004. Hardware design experiences in ZebraNet. In Proceedings of the 2nd international conference on Embedded networked sensor systems, pp. 227238. 